\title{
NANOPARTICLE TRACKING ANALYSIS OF LATEX STANDARDIZED BEADS
}

\author{
TOMASZ ŚLIWA ${ }^{\mathrm{a}, \mathrm{b}}$, MACIEJ JARZĘBSKI ${ }^{\mathrm{a}, \mathrm{b}}$, KOSMA SZUTKOWSKI $^{\mathrm{a}, \mathrm{c}}$ \\ ${ }^{a}$ Adam Mickiewicz University, Faculty of Physics, Department of Molecular Biophysics, Umultowska 85, 61-614 Poznan, Poland \\ ${ }^{b}$ NanoBioMedical Centre, Adam Mickiewicz University, 85, 61-614 Poznań, Poland. \\ ${ }^{\mathrm{c}}$ Adam Mickiewicz University, Department of Macromolecular Physics, Umultowska 85, 61-614 Poznan, Poland
}

Received March 03, 2014; accepted May 20, 2014; published online May 25, 2014

\begin{abstract}
The most popular technique for particle size characterization is the dynamic light scattering (DLS). In recent years new advanced method based on counting each single particle movement was introduced giving perspective for measurement of each component of mixture. This study presents some advantages of nanoparticle tracking analysis (NTA) method in comparison to DLS. For tests standard polystyrene beds were chosen vary diameter of 22, 61 and $150 \mathrm{~nm}$ and its mixtures. Experiments showed that the particles size resolution allows to distinguish each population in two population suspension opposed to DLS. The NTA method permits to eliminate the negative effects i.e. dust or aggregates in sample during post processing, that permits to use it in a variety of studies.
\end{abstract}

\section{INTRODUCTION}

Nanotechnology has opened new opportunities for quick application of new advanced materials. Nanoscience describes structures of the size ranging from 1 up to 100 $\mathrm{nm}$ and interactions between them. In practice, many other structures of the size under $1000 \mathrm{~nm}$ are also referred to as nanoparticles.

Polymers are one of the most commonly used materials for various medical applications. They can be easily modified to obtain target physical, chemical and structural properties. It is known that the behavior of polymers depends on the presence of foreign atoms, compounds and modifiers (i.e. plasticizers, fillers, pigments, etc. ) inserted to the carbon frame.

The connection between nanotechnology and polymer science is easy to see, especially with development of advanced material for potential and currently applied medical application. The main goal of the developing new processes of synthesis and modification is preparation of monodispersed, stable, biocompatible and biodegradable nanoparticles (Faraji \& Wipf, 2009; Panyama \& Labhasetwara, 2003, Ravichandran 2009). For biomedical applications, the factors such as particle size, polymer composition, particle hydrophobicity and surface charge, dose of particles, administration vehicle, animal species and age, fed state of the animals and use of targeting agents affect the extent of uptake of microparticles and nanostructures (O'Hagan, 1998).

Polymer nanobeads present a flexible platform for applications in diagnostics and bioseparations (Bangs-
Laboratories, 2014). One of the most intensively investigated polymers are nanosized latex spheres. There is a large number of possible modifications such as coreshell structures preparations or surface functionalisations. For the covalent immobilization of proteins, peptides, and nucleic acids, functionalized latex have been extensively investigated. For the covalent immobilization of bio structures commonly used are carboxyl or amine-functionalized polystyrene (PS) nanoparticles. For confocal microscopy, fluorescent particles are needed and available commercially as standards or could be prepared e.g. by Flow Focusing technology combined with a solvent evaporation/extraction procedure (Gañán-Calvo et al., 2006). Another option is to change the core of the PS by inserting foreign particles. For medical application, such as preparation of diagnostics test or purification of cells and biomolecules, like antibodies, nucleic acids, and polypeptides, iron oxide magnetic particles are encapsulated into PS matrix. The surfaces of encapsulated magnetic microspheres are expected to be free of iron oxide, because of the possible toxic effect on the living cell (BangsLaboratories, 2014).

Raw materials, such as commercial polystyrene microbeads, are developed by a number of alterations. Chatterjee et al. have shown that albumin microspheres revealed higher coupling efficiency with the protein and the red blood cells than the polystyrene-based microspheres (Chatterjee, Haik \& Chen, 2001). Ma et al. have proposed the synthesis of multicolor quantum dot QD-encoded microspheres, via the layer-by-layer $(\mathrm{LbL})$ 
assembly approach to detect different antigens. The method they propose avoids elaborate chemical reactions, and allows control of QD-loading and microspheres composition (Ma et al., 2007). Kanwal et al. have proposed a specific sandwich immunoassay method for human-immunoglobulinG ( $\mathrm{HIgG}$ ) using polystyrene microspheres based sandwich immunesensor with CdTe nanoparticles. The system described exhibits a linear response range of $\mathrm{HIgG}$ with an extremely low detection (Kanwal S., Traore Z. \& Su X., 2010). PS microspheres modified with b-galactosidasewere investigated for an efficient introduction into neural stem cells with no apparent toxic effect, thus providing proof-of-concept for the use of microspheres as an alternative biomolecule delivery system (Gennet et al., 2009).

As we mentioned, the final properties of the polymer particles, depend on their size. In this paper we focus on a comparison of two laser techniques used for particle size determination. Frequently used dynamic light scattering (DLS) is an ISO 13321:1996 standard. The nanoparticle tracking analysis (NTA) which is also called particle tracking or single particle tracking pretends to be a novel standard. In (Boyd et al. 2011) three techniques DLS, NTA and AFM were compared, and found out that the results are different in quantity but it is dependant to the nature of each measurement. The NTA method is used in advanced materials investigation for possible medical applications i.e. for IgG characterization (Joubert et al., 2011) and materials science such as nanocomposites (Jakubowicz, 2008).

We have chosen different sized latex standardized microbeads as the material to investigate because of their low polydispersity and wide range of biomedical application. The main goal of our study was to characterise advantages and disadvantages of each technique.

\section{MATERIALS AND METHODS}

\section{Materials}

For the test we used latex spheres with particle diameters $\left(\mathrm{d}_{\text {nominal }}\right): 22,61$ and $150 \mathrm{~nm}$. The 22 and $61 \mathrm{~nm}$ nanoparticles originated from Invitrogen made of sulfate polystyrene. The $150 \mathrm{~nm}$ particles originated from Microparticles $\mathrm{GmbH}$ and were made of polystyrene. The manufacturer concentration of $22 \mathrm{~nm}$ was $3.9 \mathrm{~g} / \mathrm{mL}, 61 \mathrm{~nm} 7.8 \mathrm{~g} / \mathrm{mL}$ and for $150 \mathrm{~nm} 10 \mathrm{~g} / \mathrm{mL}$ respectively.

\section{Nanoparticle tracking analysis}

In the study a Nanosight NS500 instrument was used, which combines DLS and NTA technique. The particle tracking measurements were based on the recorded Brownian motion. The main idea was to record video of the particle movements illuminated by the laser. The laser wavelength used in this setup was $405 \mathrm{~nm}$. The objects could be observed directly from the flow cell by the microscope objective. Also fluorescence particles could be measured, because the NS500 instrument was equipped with a fluorescence filter. The images are collected by the CCD camera and recorded. The software allows a choice of particles to be tracked, measurement of the mean square displacement of each selected particle and control of the experiment procedure i.e. cell temperature, camera sensitiveness, time of the experiment. The concentration of the sample is controlled by an automated system of two pumps for loading and diluting the sample, respectively (NanoSight Technical data, 2012).

In the NTA technique the diffusion coefficient is obtained from the analysis of the particle displacement $\mathrm{X}$ using the formula:

$$
\left\langle X^{2}\right\rangle=4 D_{N T A} t,
$$

The average self diffusion coefficient is independent of molar weight of components (equation 3).

$$
D_{N T A}=\frac{\sum D_{i} N_{i}}{\sum N_{i}},
$$

where $D_{\mathrm{NTA}}$ is the diffusion coefficient, $\mathrm{D}_{\mathrm{i}}$ and $\mathrm{N}_{\mathrm{i}}$ are diffusion coefficient and number concentrations of fraction " $i$ " respectively.

\section{Hydrodynamic radius}

Nanosight NS500 permits the use of the dynamic light scattering method (DLS) to estimate the average particle size and its polydispersity index (PdI). The DLS method is one of the most popular nondestructive methods for particle size analysis. It is also based on the particles Brownian motion, however the particles are not observed directly like in the NTA method. The fluctuations of light scattered by the particles are measured and then the autocorrelation function is calculated.

The comprehensive introduction to the DLS method is given in (Berne \& Pecora, 1976). In a light scattering experiment, the scattered light intensity fluctuates because of the Brownian motion of particles. The intensity autocorrelation function is given by equation 3 :

$$
g^{2}(\tau)=B\left[1+\beta\left|g^{1}(\tau)\right|^{2}\right],
$$

where $B$ is the baseline, $\beta$ is the coherence factor; $g^{l}(\tau)$ stands for the monodisperse particle function and is 
given by equation 5 :

$$
g^{1}(\tau)=\exp (\Gamma \tau)
$$

where $\Gamma$ is proportional to the square of scattering vector $q$ (equation 6):

$$
\Gamma=D q^{2},
$$

where $D$ is the diffusion coefficient obtained from the experiment.

Hydrodynamic radius $\left(R_{h}\right)$ is found indirectly from the diffusion coefficient given by the Stokes-Einstein equation (5).

$$
D=\frac{k_{B} T}{6 \pi \eta R_{h}},
$$

where $k_{B}$ is the Boltzmann constant and $\eta$ - the solvent viscosity, $T$ - temperature. The average diffusion coefficient from the dynamic light scattering method is given by equation (6).

$$
D_{D L S}=\frac{\sum D_{i} M_{i}^{2} N_{i}}{\sum M_{i}^{2} N_{i}},
$$

where $D_{i}, M_{i}, N_{i}$ are the diffusion coefficient, molar weight and concentration of fraction " $i$ ", respectively.

\section{EXPERIMENTS}

The testing procedure consisted of preparation of the initial water suspension of each type of microparticles $(0.2 \mu \mathrm{L}$ in $2000 \mu \mathrm{L}$ suspension) and then the NS500

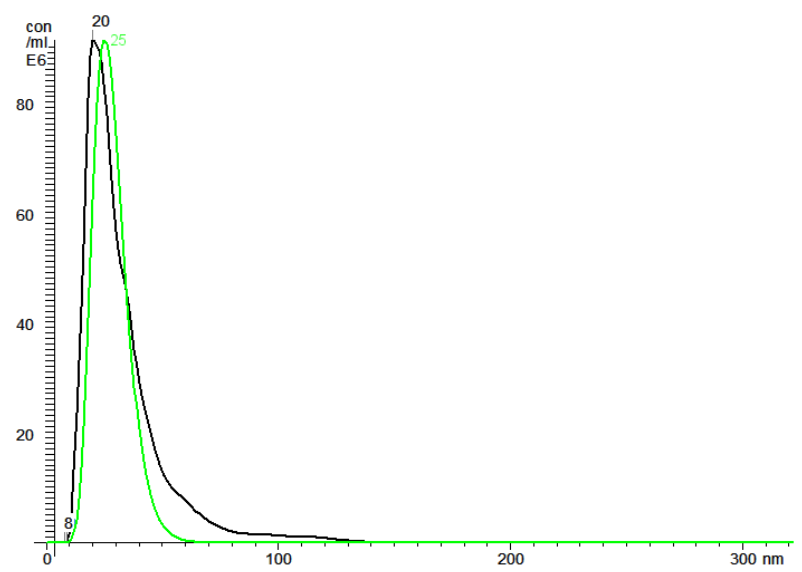

Fig. 1. Particle size distribution according to NTA and DLS (green), particles $22 \mathrm{~nm}$. instrument software was set to making dilutions of $1 \%$ of the initial suspension and $99 \%$ of the solvent. In the final concentration both NTA and DLS tests were made.

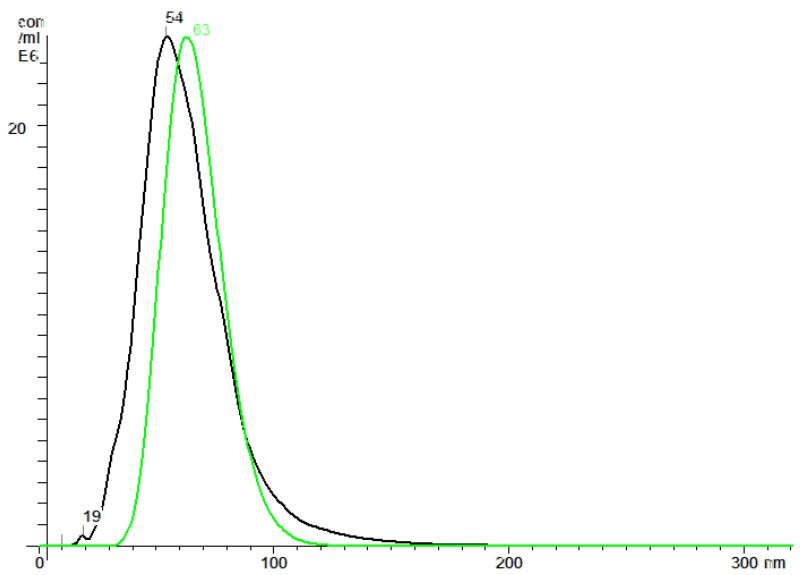

Fig. 2. Particle size distribution according to NTA and DLS (green) particles $61 \mathrm{~nm}$.

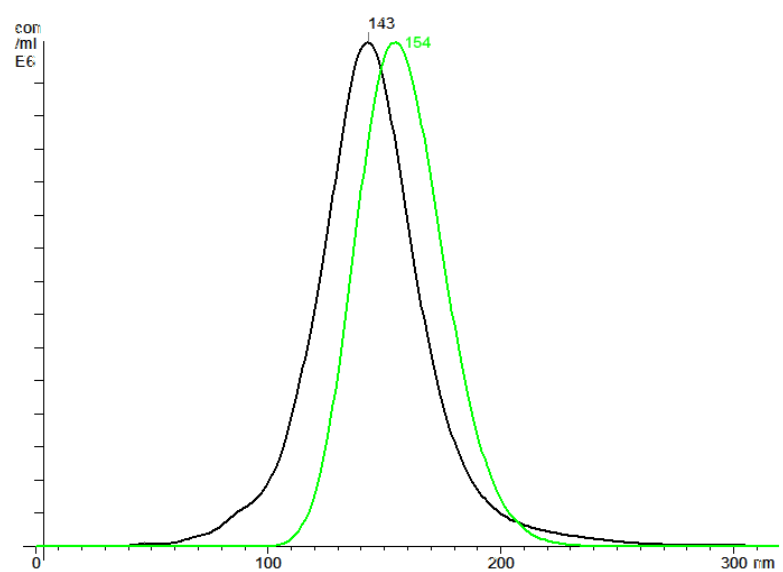

Fig. 3. Particle size distribution according to NTA and DLS , particles $150 \mathrm{~nm}$.

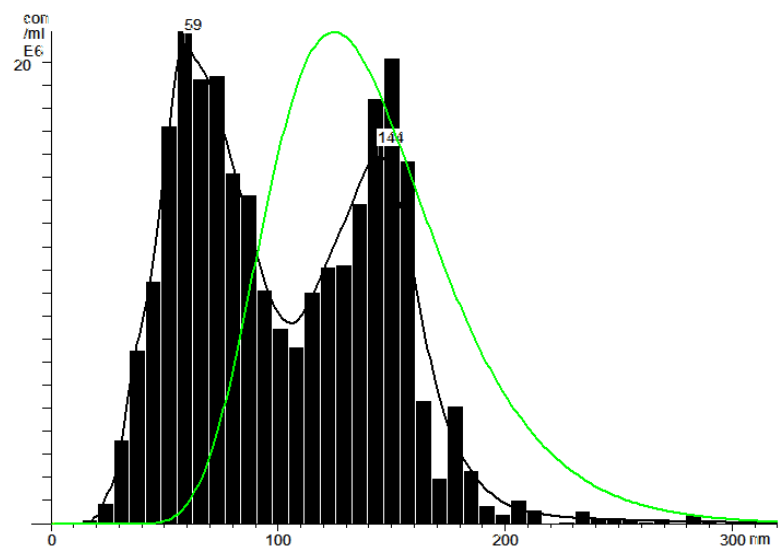

Fig. 4. Particle size distribution according to NTA and DLS (green), particles $61 \mathrm{~nm}$ and $150 \mathrm{~nm}$. 


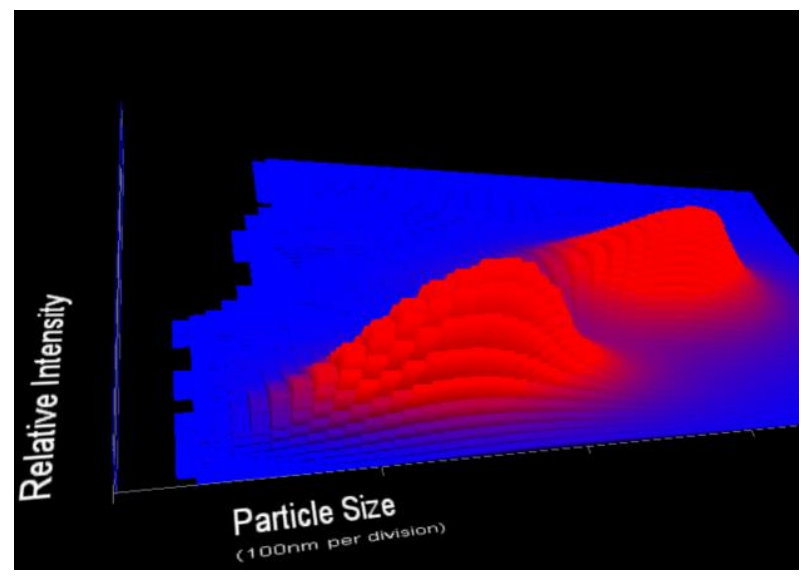

Fig. 5. Relative intensity of measured particles of $61 \mathrm{~nm}$ and $150 \mathrm{~nm}$.

\section{RESULTS AND DISCUSSION}

The aim of this study was to verify the benefits of NTA method for particle size determination versus the performance of the earlier used DLS method. The test was performed on a sample containing two populations of different size monodispersed particles. Measurement of $22 \mathrm{~nm}$ spheres are in the lower range of the instrument, and the single particles are almost invisible for the camera. It results in broad particle size distribution when compared to the results obtained from DLS (fig.1). The small size particles are more sensitive to dust that may appear in the suspension so the shape of the particle size distribution is asymmetrical. The results for $150 \mathrm{~nm}$ particles (fig.3) from NTA and DLS overlap, with a slight shift of the DLS results towards larger particles. The results obtained by NTA and DLS for $61 \mathrm{~nm}$ latex nano beads are presented in figure 2. Also the maximum of the peak obtained from DLS measurement is shifted to the right side.

In general, the DLS method is sensitive to particle size. The small amount of larger particles in the suspension scatter more light, which results in a shift of the average particle size to larger sizes and this phenomenon was confirmed by the results shown in figures 1-3. The particle size distribution obtained by DLS method are always shifted towards larger particles with respect to those following from NTA. It could be concluded that the broader the size distribution (PdI) the greater the shift in particle size, because a higher PdI corresponds to a broader particle distribution. The NTA method accurately measured the particle size, and the results it provided are consistent with the nominal particle size given by the manufacturer. The NTA method is recommended when not only the average size distribution but the modal values of the particle population distribution are to be found. Detailed results of the particle diameters (the nominal and measured by DLS method with the polydispersity indexes) are presented in Table 1. The particles of the nominal diameters $\left(\mathrm{d}_{\text {nominal }}\right)$ of $21 \mathrm{~nm}$, were measured by DLS $\left(\mathrm{d}_{\mathrm{DLS}}\right)$ and NTA to have diameters of $25 \mathrm{~nm}$ and $30 \mathrm{~nm}$, respectively. However, the modal value $\left(\mathrm{d}_{\text {mode }}\right)$ is $20 \mathrm{~nm}$ that is proximal to the nominal result. Modal value was a value of diameter that appeared most frequently in particles size distribution. The mean diameter $\left(\mathrm{d}_{\text {mean }}\right)$ was calculated as a Gaussian fit to experimental size distribution obtained from NTA. For comparison of DLS and NTA, the mean diameters were taken to account. Nevertheless, the calculated mode particle size is more valuable for the investigation of biostructures or systems for potential medical applications. Nanoparticles used for medical applications should have well-defined size and low polydispersity. They are better represented by the mode diameter calculated by the software. A mixture of particles whose nominal diameters were $61 \mathrm{~nm}$ and $150 \mathrm{~nm}$ was characterized by NTA and DLS (fig.4). By the DLS method, the average particle size and its distribution calculated using the instrument software were between the nominal particles sizes (measured as $59 \mathrm{~nm}$ and $144 \mathrm{~nm}$ ) and was $124 \mathrm{~nm}$. This value was obtained on the basis of monoexponental fit corresponding to the presence of a single population of particles as the NTA software is set up to calculate the monomodal distribution of particle size. The results from NTA show the presence of two populations of particles of different sizes. Also the difference in the intensity makes the smaller size $61 \mathrm{~nm}$ particles "hidden" behind the $150 \mathrm{~nm}$ particles for the DLS technique. Further analysis of DLS data obtained by NS500 instrument might be performed on the exported data for example in CONTIN that potentially will allow getting information about both populations in the sample tested.

Table 1. Particle sizes: from manufacturer $\left(\mathrm{d}_{\text {nominal }}\right)$, form DLS $\left(\mathrm{d}_{\mathrm{DLS}}\right)$, mean value form NTA distribution $\left(\mathrm{d}_{\text {mean }}\right)$, modal value from NTA distribution $\left(\mathrm{d}_{\text {mode }}\right)$, polydispersity index (PdI).

\begin{tabular}{lllll}
\hline $\mathrm{d}_{\text {nominal }}$ & $\mathrm{d}_{\text {DLS }}$ & PdI & $\mathrm{d}_{\text {mean }}$ & $\mathrm{d}_{\text {mode }}$ \\
\hline $21 \mathrm{~nm}$ & $25 \mathrm{~nm}$ & 0.42650 & $30 \mathrm{~nm}$ & $20 \mathrm{~nm}$ \\
$61 \mathrm{~nm}$ & $63 \mathrm{~nm}$ & 0.13861 & $64 \mathrm{~nm}$ & $54 \mathrm{~nm}$ \\
$150 \mathrm{~nm}$ & $154 \mathrm{~nm}$ & 0.05325 & $153 \mathrm{~nm}$ & $148 \mathrm{~nm}$ \\
\hline
\end{tabular}

Similar tests were performed by (Filipe V., Hawe A. \& Jiskoot W., 2010), who used particles sized 60-1000 nm to test the capabilities of particle tracking-based instrument. These authors also concluded that the presence of larger particles in the suspension has no influence on the accuracy of the NTA method, but the smaller particles might not have been well detected as was the case in measurement of the $22 \mathrm{~nm}$ latex particles reported in this article. 


\section{CONCLUSION}

The NTA-based method is useful to measure the size of particles population. As mentioned above, in the NTA method the particles individually tracked and on the basis of the track recorded the mean square displacement is calculated, so also the particle size distribution, regardless of the number of differing in size populations. The precision of measurement for mixtures of different size particles is higher than in DLS, whilst this method does not handle with the differences of scattered light intensity obtained from different size particles. The whole process of the sample dilution and instrument setup is easy for the operator. The potential mistakes in the measurement parameter setup may be corrected in the post-processing procedure.

\section{ACKNOWLEDGMENTS}

The authors wish to thank Adam Patkowski and Jacek Gapiński from the Adam Mickiewicz University, Faculty of Physics, for many fruitful discussions.

Tomasz Śliwa thanks the NanoBioMedical Centre in Poznan, Adam Mickiewicz University and Faculty of Physics where Ph.D. thesis is realised under the project co-financed by European Union, programme Human Capital National Cohesion Strategy funded by European Social Fund. Maciej Jarzębski, thanks the Foundation for Polish Science and NanoBioMedical Centre in Poznan, Adam Mickiewicz University, Faculty of Physics as his work was supported by the International $\mathrm{PhD}$ Projects Programme of Foundation for Polish Science operated within the Innovative Economy Operational Programme (IE OP) 2007-2013 within European Regional Development Fund. Kosma Szutkowski thanks the PBS programme "Nanomaterials and their application to biomedicine" funded by the National Centre for Research and Development.

\section{REFERENCES}

Bangs Laboratories, Inc. (9.02.2014) http://www.bangslabs.com.

Berne B.J., Pecora R., (1976) Dynamic Light Scattering: with Applications to Chemistry, Biology, and physics, Wiley, New York.
Boyd RD, Pichaimuthu SK., Cuenat A. (2011) New approach to inter-technique comparisons for nanoparticle size measurements; using atomic force microscopy, nanoparticle tracking analysis and dynamic light scattering. Colloids and Surfaces A: Physicochemical and Engineering Aspects, 387, 35-42.

Chatterjee J., Haik Y., Chen C. J. (2001) Modification and characterization of polystyrene-based magnetic microspheres and comparison with albumin-based magnetic microspheres, Journal of Magnetism and Magnetic Materials, 225, 21-29.

Faraji A., Wipf P. (2009) Nanoparticles in cellular drug delivery. Bioorganic \& Medicinal Chemistry, 17, 29502962.

Filipe V., HaweA., Jiskoot W., (2010) Critical Evaluation of Nanoparticle Tracking Analysis (NTA) by NanoSight for the Measurement of Nanoparticles and Protein Aggregates, Pharmaceutical Research, 27, 796-810.

Gañán-Calvo A. M., Martín-Banderas L, González-Prieto R., Rodríguez-Gil A., Berdùn-Álvarez T., Cebolla A., Chávez S., Flores-Mosquera M., (2006) Straightforward production of encoded microbeads by Flow Focusing: Potential applications for biomolecule detection, International Journal of Pharmaceutics, 324, 19-26.

Gennet N., Alexander L. M., Sánchez-Martín R. M., Behrendt J. M., Sutherland A. J., Brickman J. M., Bradley M. \& Li M (2009) Microspheres as a vehicle for biomolecule delivery to neural stem cells, New Biotechnology, 25 (6), 442-449.

Jakubowicz J. (2008) Particle analysis and properties of mechanically alloyed $\mathrm{Nd}_{16} \mathrm{Fe}_{76-\mathrm{x}} \mathrm{Ti}_{\mathrm{x}} \mathrm{B}_{8}$, Superlattices and Microstructures, 43, 315-323.

Joubert MK, Luo Q, Nashed-Samuel Y, Wypych J, Narhi LO. (2011) Classification and characterization of therapeutic antibody aggregates, $J$ Biol Chem., 286, 25118-2513.

Kanwal S., Traore Z. \& Su X. (2010) Polystyrene microspheres based sandwich immunosensor using CdTe nanoparticles amplification and ultrasensitive flow-injection chemiluminescence detection, Colloids and Surfaces B: Biointerfaces, 81, 549-554.

Ma Q., Wang X., Li Y., Shi Y. \& Su X. (2007) Multicolor quantum dot-encoded microspheres for the detection of biomolecules, Talanta, 72, 1446-1452.

NanoSight (2012) Technical data: NTA 2.3 Analytical Software, Operating Manual P554J, NanoSight Ltd., Minton Park, Amesbury, Wiltshire SP4 7RT, UK.

O'Hagan D. T. (1998) Microparticles and polymers for the mucosal delivery of vaccines, Advanced Drug Delivery Reviews, 34, 305-320.

Panyama J. \& Labhasetwara V., (2003) Biodegradable nanoparticles for drug and gene delivery to cells and tissue Advanced Drug Delivery Reviews, 55, 329-347.

Ravichandran R. (2009) Nanotechnology-Based Drug Delivery Systems, Nanobiotechnol., 5, 17-33. 